\title{
Giant serous microcystic adenoma of the pancreas safely resected after preoperative arterial embolization
}

\author{
HIDEHIRO TAJIMA ${ }^{1}$, TETSUO OHTA ${ }^{1}$, HIROHISA KITAGAWA ${ }^{1}$, HIROYUKI SHINBASHI ${ }^{1}$, \\ ATSUSHI HIROSE ${ }^{1}$, SEISHO SAKAI ${ }^{1}$, ISAMU MAKINO ${ }^{1}$, HIRONORI HAYASHI ${ }^{1}$, HISATOSHI NAKAGAWARA ${ }^{1}$, \\ ICHIRO ONISHI $^{1}$, HIROYUKI TAKAMURA ${ }^{1}$, ITASU NINOMIYA ${ }^{1}$, SACHIO FUSHIDA ${ }^{1}$, \\ TAKASHI TANI ${ }^{1}$, TAKASHI FUJIMURA ${ }^{1}$, MASATO KAYAHARA ${ }^{1}$, WATARU KODA ${ }^{2}$ and OSAMU MATSUI ${ }^{2}$ \\ Departments of ${ }^{1}$ Gastroenterologic Surgery and ${ }^{2}$ Radiology, Division of Cancer Medicine, \\ Graduate School of Medicine Science, Kanazawa University, Kanazawa, Japan
}

Received April 19, 2010; Accepted May 24, 2010

DOI: 10.3892/ol_00000148

\begin{abstract}
Serous microcystic adenomas are rare and account for $1-2 \%$ of all exocrine pancreatic tumors and $25 \%$ of all pancreatic cystic neoplasms. Recently, with advances in imaging techniques, these adenomas have been identified at an increasing frequency. A 63-year-old woman visited her doctor in 1999 due to a gastric deformity detected by upper gastrointestinal endoscopy. An abdominal computed tomography scan revealed a cystic lesion measuring $6.0 \mathrm{~cm}$ in diameter, resulting in a diagnosis of serous microcystic adenoma of the pancreatic head. During follow-up, the tumor increased steadily in size, measuring $6.0 \mathrm{~cm}$ in diameter in 1999 and $13.0 \mathrm{~cm}$ in 2008, while remaining asymptomatic throughout this period of time. The risk of malignant transformation appears to be low even over the long-term. However, some cases of malignant transformation to serous cystadenocarcinoma have recently been reported. In this case, assessment of the relationship between the tumor and adjacent vascular structures, such as massive drainage vein development on the surface or tumor flow into the portal and superior mesenteric veins and the celiac and superior mesenteric arteries, was critical for determining tumor resectability. The risk of massive intra-operative hemorrhage was felt to be considerable, given the extent of the veins on the surface of the tumor, as well as the size and location of the primary pancreatic mass. Therefore, preoperative embolization of the tumor-feeding arteries arising from the celiac axis (gastroduodenal, splenic and dorsal pancreatic arteries) was performed. Tumor resection with pancreaticoduodenectomy was performed without a blood transfusion, with an estimated blood loss of $570 \mathrm{ml}$. The final pathology confirmed the diag-
\end{abstract}

Correspondence to: Dr Hidehiro Tajima, 13-1 Takaramachi, Kanazawa 920-8641, Japan

E-mail: hidetaji@staff.kanazawa-u.ac.jp

Key words: serous microcystic adenoma, giant tumor, hypervascular tumor, pancreas, preoperative arterial embolization nosis of serous microcystic adenoma. The patient is currently alive and disease-free. Preoperative partial embolization of the tumor feeding arteries and intra-operative resection of the right gastric and inferior pancreatoduodenal arteries, allowed the tumor blood supply to be arrested without preoperative tumor necrosis. Subsequently, intraoperative blood loss was reduced. Preoperative partial embolization of the feeding arteries is useful for the resection of hypervascular large tumors of the pancreas.

\section{Introduction}

Serous microcystic adenomas are rare and account for $1-2 \%$ of all the exocrine pancreatic tumors and $25 \%$ of all pancreatic cystic neoplasms. Due to advances in imaging techniques, these adenomas have been identified at an increasing frequency. The tumors usually occur in the elderly in the seventh to eighth decade of life (range 34-91 years; mean age 66) (1,2). A female preponderance has been noted (70\% of the tumors occur in women). According to the literature, these tumors range in size from $1-26 \mathrm{~cm}$, with an average of $6-10 \mathrm{~cm}(1,2,3)$. Since serous cystic adenomas are considered to be benign tumors with almost no potential for malignant transformation, surgery is usually not recommended. However, recent reports have suggested a small but finite risk of malignancy for serous cystic neoplasms of the pancreas (4). In this case, assessment of the relationship between the tumor and adjacent vascular structures, such as the massive drainage veins on the surface or tumor flow into the portal and superior mesenteric vein (SMV) as well as the celiac and superior mesenteric arteries (SMA), was considered to be critical for the preoperative determination of tumor resectability. The risk of massive intraoperative hemorrhage was felt to be considerable, given the extent of the veins on the surface of the tumor, as well as the size and location of the primary pancreatic mass.

In this study a case of large and hypervascular serous microcystic adenoma of the pancreatic head was successfully resected with the preoperative embolization of the feeding arteries. 

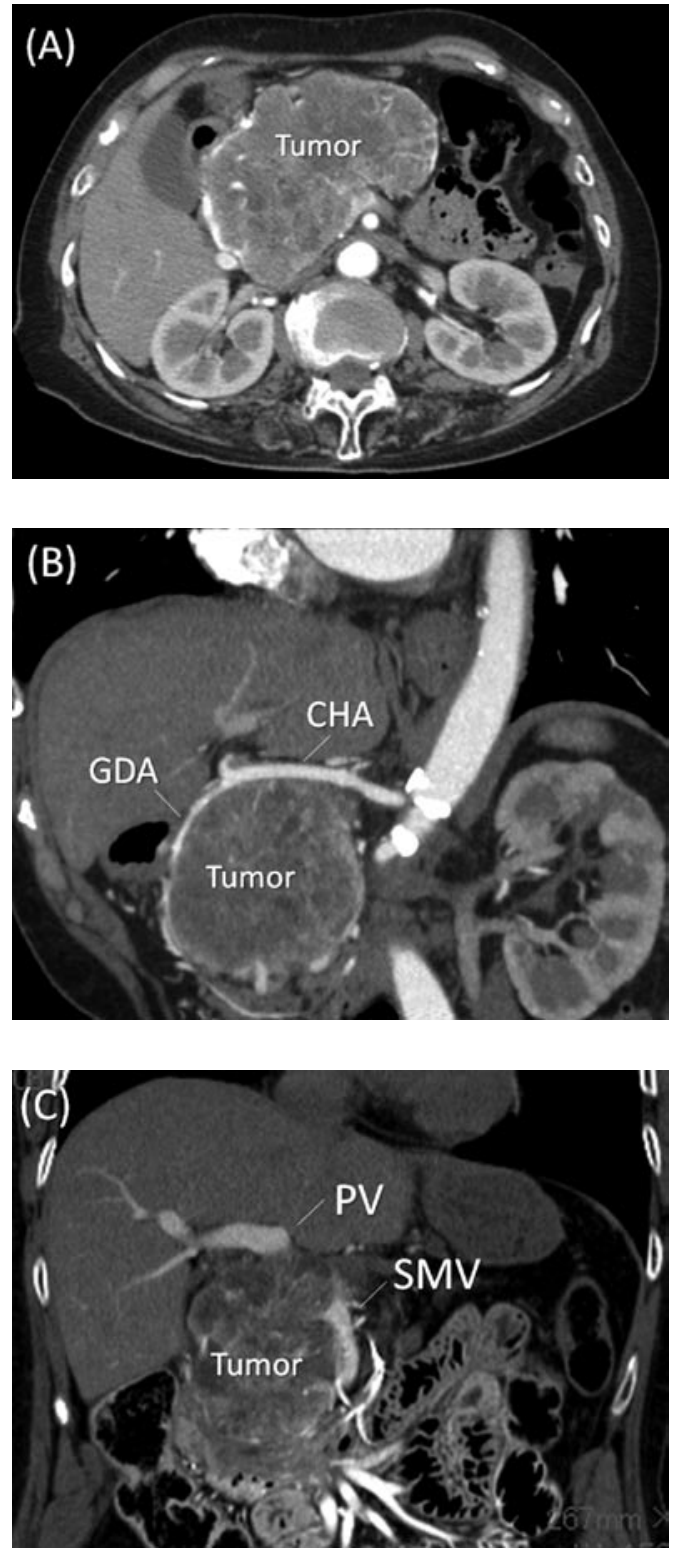

Figure 1. Multi-detector row (MD) computed tomography scan. (A) A large hypervascular tumor measuring $13.0 \mathrm{~cm}$ in diameter at the head of the pancreas is shown. (B) In the arterial phase, the common hepatic artery (CHA) and gastroduodenal artery (GDA) are stretched widely across the surface of the tumor. (C) In the portal phase, the tumor arising from the pancreatic head showed posterior compression on the portal (PV) and superior mesenteric veins (SMV).

\section{Case report}

A 63-year-old woman visited her general practitioner in 1999 owing to a gastric deformity detected by routine upper gastrointestinal endoscopy. An abdominal computed tomography scan revealed a cystic lesion measuring $6.0 \mathrm{~cm}$ in diameter, and the tumor was diagnosed as a serous microcystic adenoma of the pancreatic head. During the follow-up, the tumor increased steadily in size, measuring $6.0 \mathrm{~cm}$ in diameter in 1999 and $13.0 \mathrm{~cm}$ in 2008, while remaining asymptomatic throughout this time period. The risk of malignant transformation is believed to be low even in the long-term (5). However, some cases of malignant transformation to serous cystadenocarcinoma have been reported (4). Since the tumor was
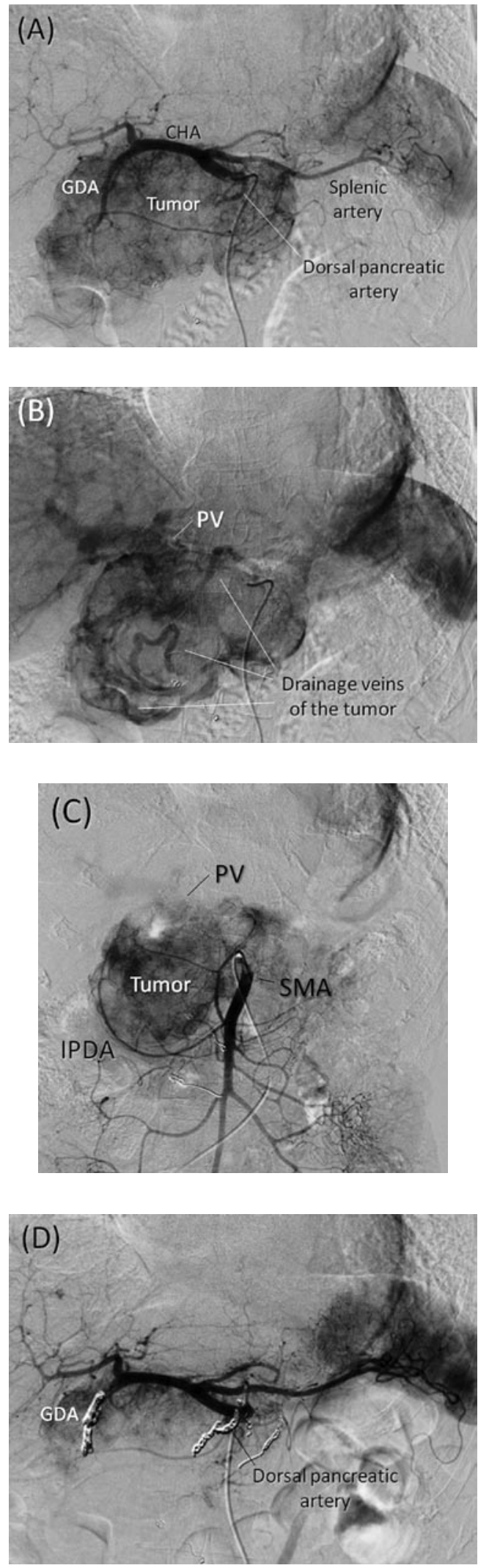

Figure 2. Angiography and preoperative embolization. (A) Early phase of the celiac axis arteriography showing feeding arteries to the tumor arising from arteries including the gastroduodenal, right gastric, splenic and dorsal pancreatic arteries. (B) Delayed phase of celiac axis arteriography showing numerous drainage veins on the surface of the tumor flowing into the PV. (C) Superior mesenteric artery (SMA) angiography showing the main feeding arteries to the inferior aspect of the tumor supplied by the inferior pancreatoduodenal artery (IPDA). (D) Preoperative embolization of the gastroduodenal, splenic and dorsal pancreatic arteries. Interlock embolization coils from the celiac axis was performed. 


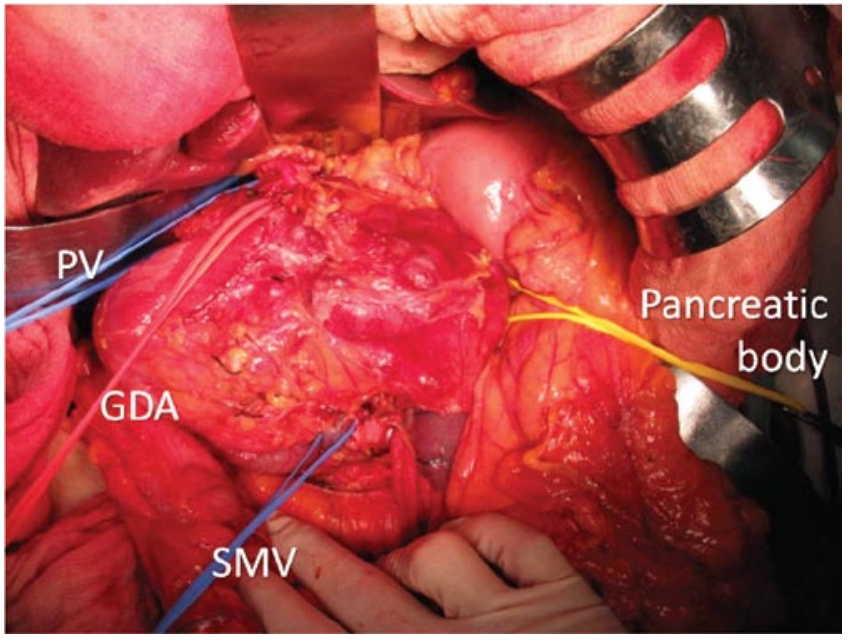

Figure 3. Laparotomy shows a large multicystic tumor arising from the head of the pancreas. A red vascular tape is looped under the gastroduodenal artery (GDA). Blue vascular tapes are looped under the PV and SMV at the upper and lower sides of the tumor, and a yellow vascular tape is looped under the transection line of the pancreatic body.

large, the patient was considered to be at risk of developing obstructive jaundice, duodenal stenosis, portal thrombosis or rupture of the tumor, and a tumor resection was performed. On multi-detector row (MD) computed tomography scan (Fig. 1) and angiography (Fig. 2), numerous feeding arteries (including gastroduodenal, right gastric, splenic, dorsal pancreatic and inferior pancreatoduodenal arteries) were found to supply blood to the tumor, and the common hepatic artery was stretched widely across the upper surface of the tumor. Moreover, several enlarged draining veins were found on the surface of the tumor, and drainage of these veins into the portal vein (PV) and SMV was observed. In the current case, although resection was deemed to be feasible, the risk of massive intraoperative hemorrhage was felt to be considerable. Therefore, preoperative embolization of the tumor-feeding arteries from the celiac axis (gastroduodenal, splenic and dorsal pancreatic arteries) was performed, with the tumor resection performed on the following day.

Laparotomy (Fig. 3) showed a large multicystic tumor arising from the pancreatic head, with numerous vessels on its surface. The distal duodenum or proximal jejunum at the ligament of Treitz was torn from the retroperitoneum, and the SMA was identified. The root of the inferior pancreatoduonenal (IPDA) was detected and the vessel was resected. The right gastric and gastroepiploic arteries were then resected. At this point, owing to preoperative embolization, the blood supply to the tumor was completely arrested and the tumor had a slightly deflated appearance. As a result, the common and proper hepatic arteries that had been in tight contact with the superior surface of the tumor were detached and the gastroduodenal artery was resected. The pancreatic body was cut at the left side of the tumor with the requisite surgical margin. While we attempted to preserve the PV, splenic vein $(\mathrm{SpV})$ and SMV, several drainage veins on the surface of the tumor were found to drain into the SMV and PV, and the tumor was tightly adherent to the SMV and PV. Therefore, the SMV-PV was resected and reconstructed by end-to-end anastomosis.
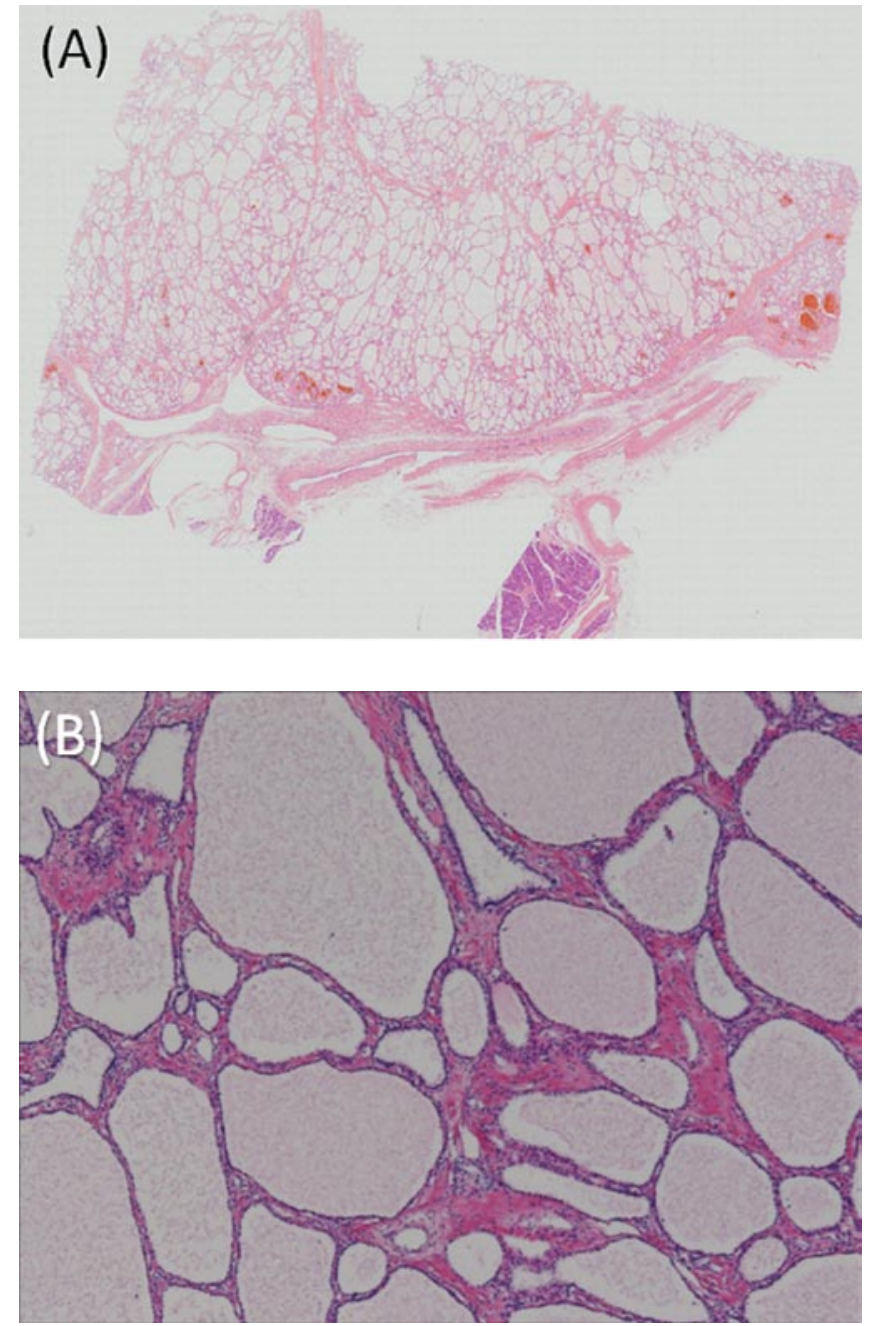

Figure 4. Microscopic features of the tumor. (A) The tumor is composed of multiple cysts lined with a single layer of cuboidal epithelium without atypia. H.E. Loupe. (B) Preoperative tumor embolization did not produce any changes, such as necrosis, that may interfere with visualization of the pathological features of the tumor. H.E. $\mathrm{x} 40$.

The estimated blood loss was $570 \mathrm{ml}$. The tumor surgery with pancreaticoduodenectomy was performed without a blood transfusion. The final pathology confirmed the diagnosis of serous microcystic adenoma (Fig. 4). Pathological examination of the resected specimen revealed arterial thrombosis due to the preoperative tumor embolization. However, no ischemic or necrotic changes occurred in the tumor. The postoperative course was uneventful, and the patient is currently alive and disease-free. The patient gave her informed consent, and consent and approval for the study was obtained by the Ethics committee.

\section{Discussion}

Cystic pancreatic neoplasms are uncommon. Differentiation between serous cystic tumors and mucinous neoplasms is crucial, due to the radically different biological characteristics of the two types of neoplasms (6,7). Mucinous cystic neoplasms should always be resected due to their premalignant nature and strong tendency towards malignant transformation (7). Management of serous tumors is more controversial since 
serous microcystic adenomas are considered to be tumors with almost no potential for malignant transformation. Thus, surgery is usually not recommended. However, during the follow-up of our case, the tumor increased steadily in size, from $6.0 \mathrm{~cm}$ to $13.0 \mathrm{~cm}$ in diameter from 1999 to 2008 . The risk of malignant transformation has been reported to be low, even in the long-term. However, some cases of malignant transformation to serous cystadenocarcinoma were recently reported (4). A case of serous cystic adenoma that increased in size, over a follow-up period of more than 20 years, eventually producing duodenal and colonic stenosis and portal hypertension was reported in 2007 (5). If these symptoms were to appear and surgery was to become necessary in the future, the patient would be much older. Therefore, tumor resection was performed.

In the current case, although resection was deemed to be feasible, the risk of massive intraoperative hemorrhage was felt to be considerable given the extent of the feeding arteries and massive drainage veins, as well as the size and location of the primary pancreatic mass. Preoperative arterial embolization was previously shown to be a safe and efficacious tumor decompression technique $(8,9,10)$. On the other hand, embolization of the feeding arteries has also been reported to cause ischemia, necrosis, inflammation and angiogenesis of the tumor. Therefore, arterial embolization was performed on the day prior to surgery for some of the arteries that were located behind the tumor and were potentially difficult to approach during surgery.

To improve prognosis, radical pancreaticoduodenectomy is usually performed and involves wide lymph node dissection and complete removal of the extrapancreatic nerve plexus of the SMA for patients with carcinoma of the pancreatic head $(11,12,13)$. Additionally, SMA is approached from the distal duodenum to proximal jejunum at the ligament of Treitz in front of the vena cava, behind the anterior renal (subperitoneal) fissure. We approached the SMA and resected the IPDA using the same technique as that during surgery for cancer of the pancreatic head. This paraduodenal approach allowed for early evaluation of the SMA. Preoperative partial embolization of the tumor-feeding arteries and intraoperative resection of the IPDA and right gastric artery resulted in the arrest of the tumor blood supply without preoperative tumor necrosis. Thus, blood loss was reduced.

Advances in imaging techniques have led to the identification of serous microcystic adenomas of the pancreas. The excellent prognosis associated with serous microcystic adenoma justifies an aggressive approach to surgical resection, even in older patients, especially since major pancreatic resections are now performed with very low mortality and morbidity rates at leading centers around the world. In conclusion, preoperative partial embolization of the feeding arteries is useful for resection of hypervascular large tumors of the pancreas. Only by utilizing multi-modality imaging, interventional radiology techniques and surgery can these complex patients be managed successfully.

\section{References}

1. Tampi C, Mullerpatan P, Shah R, Jagannath P and Zimmermann A: Microcystic serous cystadenoma of the pancreas: a case report of two cases with one of diffuse presentation. Pancreatology 6: 248-253, 2006.

2. Vernadakis S, Kaiser GM, Christodoulou E, Mathe Z, Troullinakis M, Bankfalvi A and Paul A: Enormous serous microcystic adenoma of the pancreas. J Pancreas 10: 332-334, 2009.

3. Omeroglu A, Paner GP, Ciesla MC and Harman G: Serous microcystic adenoma of the pancreas. Arch Pathol Lab Med 125: 1613-1614, 2001.

4. King JC, Ng TT, White SC, Cortina G, Reber HA and Hines OJ: Pancreatic serous cystadenocarcinoma: a case report and review of the literature. J Gastrointest Surg 13: 1864-1868, 2009.

5. Schulz HU, Kellner U, Kahl S, Effenberger O, Asperger W, Lippert $\mathrm{H}$ and Röcken C: A giant pancreatic serous microcystic adenoma with 20 years follow-up. Langenbecks Arch Surg 392: 209-213, 2007.

6. Box JC and Douglas HO: Management of cystic neoplasms of the pancreas. Am Surgeon 66: 495-501, 2000.

7. Sarr MG, Kendrick ML, Nagomey DM, Thompson GB, Farley DR and Farnell MB: Cystic neoplasms of the pancreas: benign to malignant epithelial neoplasms. Surg Clin North Am 81: 497-509, 2001.

8. Adams DB, Mauterer DJ, Vujic IJ and Anderson MC: Preoperative control of splenic artery inflow in patients with splenic venous occlusion. South Med J 83: 1021-1024, 1990.

9. Umeda Y, Yagi T, Sadamori H, et al: Preoperative proximal splenic artery embolization: a safe and efficacious portal decompression technique that improves the outcome of liver donor liver transplantation. Transpl Int 20: 947-955, 2007.

10. Joyce DL, Hong K, Fishman EK, Wisell J and Pawlik TM: Multi-visceral resection of pancreatic VIPoma in a patient with sinistral portal vein hypertension. World J Surg Oncol 6: 80-86, 2008.

11. Nagakawa T, Kurachi M, Konishi K, et al: Translateral retroperitoneal approach in radical surgery for pancreatic carcinoma. Jpn J Surg 12: 229-233, 1982.

12. Nagakawa T, Nagamori M, Futakami F, et al: Result of extensive surgery for pancreatic carcinoma. Cancer 77: 640-645, 1996.

13. Noto M, Miwa K, Kitagawa H, et al: Pancreas head carcinoma. Frequency of invasion to soft tissue adherent to the superior mesenteric artery. Am J Surg Pathol 29: 1056-1061, 2005. 